\title{
Analysis of the efficacy of a psychotherapeutic program to improve the emotional status of caregivers of elderly dependent relatives
}

\author{
Javier López ${ }^{\text {a* }}$ and Maria Crespo ${ }^{\text {b }}$ \\ ${ }^{a}$ Department of Psychology, University San Pablo CEU, Madrid, Spain, ${ }^{b}$ Faculty of de Psychology. \\ Universidad Complutense of Madrid, Madrid, Spain
}

(Received 7 November 2007; final version received 4 June 2008)

\begin{abstract}
Objectives: This study examined the long-term impact of a psychotherapeutic cognitive-behavioral program with two intervention formats (traditional weekly sessions (TWS) and minimal therapist contact (MTC)) in caregivers who suffered from emotional problems due to caring for elderly dependent relatives.

Method: The 86 participants, who lived with the older persons at home, were randomized into one of the two intervention formats. The individual treatment program was earried out during an 8-week interval. Measures of anxiety, depression, burden, coping, social support, and self-esteem were analyzed at pre- and post-treatment, and at 1-, 3-, 6-, and 12-month follow-ups.

Results: Significant effects were found in the expected direction in most of the measures analyzed. The participants in the intervention reduced significantly their levels of anxiety, depression, and burden, and they improved the levels of problem-focused coping, social support, and self-esteem. The two intervention formats had different evolutions, with better effects in the TWS format, especially at the first post-test measurements, but the differences tended to decrease over time.

Conclusion: These data suggest that individual psychotherapeutic interventions with caregivers are efficient to rednce their emotional problems, and that this effect is mediated by improvement both in their appraisal of the situation and in their personal resonrces.
\end{abstract}

Keywords: Caregiving; psychological treatment; randomized clinical trial; anxiety; depression

\section{Introduction}

Family members play an essential role in the care of elderly dependent people. They carry out the role of caregivers of their relatives for long periods of time, which places them in a highly vulnerable situation (Brodaty, Green, \& Koschera, 2003; Sörensen, Pinquart, \& Duberstein, 2002). Although caregivers may experience some positive aspects (López, López-Arrieta, \& Crespo, 2005), to take on the role of caregiver is an important source of stress, which increases the risk of suffering from diverse physical problems, as well as important emotional alterations, especially anxiety and depression (Cuijpers, 2005; Schulz, O'Brien, Bookwala, \& Fleissner, 1995).

To counteract these negative effects, interventions developed to date have been, in addition to fairly numerous, very heterogeneous, both in their goals and in their content and format (Brodaty et al, 2003; Cooke, McNally, Mulligan, Harrison, \& Newman, 2001; Knight, Lutzky, \& Macofsky-Urban, 1993; Pusey \& Richards, 2001; Sörensen et al., 2002). However, these interventions can generally be grouped into: formal support services, educational programs, mutual support groups, and psychotherapeutic interventions; although the conjoint use or combination of several of these options is also common (Gallagher, 1985).
An evolution in the content of the interventions has been observed in the literature on caregivers: from the presentation of information in the oldest works, to providing strategies and tools to help solve concrete problems in the most recent ones (López \& Crespo, 2007). Most of the psychotherapeutic programs have been of a cognitive-behavioral orientation. These interventions usually include training in the control of arousal, cognitive restructuring, problem-solving training, and increase in pleasant activities, to which other elements can be added (e.g., self-instructions, assertiveness training, (...). Diverse techniques are used to administer them, (group discussion, role-playing, reading, audiovisual material,...), and there seems to be no similarity among the various interventions (López \& Crespo, 2007; Sörensen et al, 2002). Treatments aimed at reducing the emotional problems suffered by a large part of caregivers, which have achieved small or moderate results (Knight et al., 1993), but individual psychotherapeutic treatments seem to obtain better results than other kinds of interventions (Knight et al, 1993; López \& Crespo, 2007),

Although caregivers may benefit from this kind of interventions, especially with regard to depression (Sörensen et al, 2002), family members who care for the elderly at home are difficult to access. Many of them are not interested in participating in programs to

*Corresponding author. Email: jlopezm@ cen.es 
help them to improve their situation, and others, despite their interest, have severe difficulties that prevent them from becoming involved. In fact, in general, they show little initiative in using the available resources. Therefore, it seems essential to adapt the format of the interventions to caregivers' specific needs and characteristics (especially, to the scarce amount of free time and their burden of work). In this sense, the data indicate that long interventions, though effective, generate a large number of dropouts. It appears important to offer short intervention programs that do not add more stress, which are already endured by caregivers (Knight et aL, 1993; López \& Crespo, 2007 ).

This suggests that a good alternative would be to develop minimal therapist contact interventions, which would avoid the high cost for burdened caregivers of having to travel across town to be interviewed by the therapist, and which have shown promising results in the treatment of emotional problems (Gould \& Clum, 1993; Rowan \& Storey, 2002). This kind of interventions usually uses reduced contact with patients, either face-to-face, by phone, or by other means (e.g., mail, Internet), during which caregivers are provided with written or audiovisual material that they work on, and that is subsequently reviewed. In any case, the patients are the most active agents of the intervention because they are acquiring new knowledge and skills to cope with their problems, mainly at home and not in the therapist's office.

Some of the advantages of minimal therapist contact interventions are: (a) patients' greater involvement in the acquisition of skills, (b) reduced costs for the patient and the therapist, (c) higher accessibility of treatments (e.g., rural zones), (d) reduction of patients' fear and apprehension toward treatment, and (e) higher flexibility (e.g., better adapted to patients' schedules). But it should not be forgotten that these treatments also have some disadvantages, such as: (a) the need for patients' higher involvement in the interventions, (b) the need for patients' higher motivation, (c) the danger of patients' lack of adherence to treatment, and (d) the inappropriateness of the format for certain patients (e.g., functional illiteracy, presence of suicidal ideation) (Rowan \& Storey, 2002). Furthermore, caregivers need intensive interventions and they must have time to interiorize and put the strategies and tools proposed during the intervention into practice (Sörensen et al., 2002).

Most of the minimal therapist contact interventions carried out with caregivers have consisted of listening to their troubles and offering them, by phone, information that may be of use (Chang, 1999; Davis, Burgio, Buckwalter, \& Weaver, 2004; Eisdorfer et al., 2003; Mahoney, Tarlow, \& Jones, 2003). But mere phone attention to caregivers does not generally reduce the emotional variables analyzed. In contrast, the incorporation of the new technologies is resulting in the creation of new pioneer interventions by Internet (Beauchamp, Irvine, Seeley, \& Johnson, 2005; Marziali \& Donahue, 2006; White \& Dorman, 2000) that show promising results at post-treatment. However, followup studies are needed to determine whether or not the immediate results are maintained, and whether new fruits of the progressive assimilation of their contents emerge.

As indicated by Brodaty et al. (2003), despite the efforts already made, it is still essential to continue investigating the efficacy of the various treatments proposed to help caregivers deal with the problems of stress resulting from their situation. Thus, the purpose of this investigation was to explore the efficacy of a brief individual psychological intervention, with two intervention formats, one with traditional weekly sessions (TWS) and the other with minimal therapist contact (MTC), aimed at stress management for the people who must care for an elderly dependent at home. In its two formats, the main goal of the intervention was to reduce caregivers' levels of emotional problems (anxiety and depression).

\section{Methods}

\section{Participants}

The participants came from various Health Centers, Municipal Social Services, and Family Associations of the Community of Madrid (Spain). The caregivers met the following inclusion criteria: (a) they were caring for a noninstitutionalized older person (over 60 years of age); (b) the elderly person was dependent, that is, he/she needed supervision or help to perform one or more of the activities assessed by the Katz Index of Activities of Daily Living (ADL; Katz, Ford, Moskowitz, Jackson, \& Jaffe, 1963); (c) the caregiver was the main person responsible for the care of the older person; (d) they lived permanently in the same domicile with the older person; (e) they had been caring for their relative for at least 6 months; (f) they were not receiving any other individual or group psychological treatment; and (g) they presented emotional problems as determined by scores over 9 in the Beck Depression Inventory (BDI; Beck, Rush, Shaw, \& Emery, 1979) or scores over 7 in the Anxiety Subscale of the Hospital Anxiety and Depression Scale (HAD-A; Zigmond \& Snaith, 1983). We decided to include only caregivers who presented emotional problems because the treatment was aimed at overcoming these problems and therefore, would be of no interest to people whose emotional status or anxiety levels were unaffected, despite their caring for a relative.

A total of 123 caregivers were assessed for their possible participation in this investigation; $14(11 \%)$ did not meet the inclusion criteria, $18(15 \%)$ declined to participate for various reasons, and $5(4 \%)$, due to temporary problems, could not be treated according to the intervention protocol designed for this investigation. Therefore, the total sample was made up of 86 caregivers. 


\section{Intervention}

Intervention overview

In order to adapt the intervention to the specific characteristics of the caregivers, it was based both on the predictors of poorer emotional status and a higher level of anxiety among the caregivers (Crespo, López, \& Zarit, 2005), and on the bibliographic review of intervention studies with caregivers of elderly dependents (López \& Crespo, 2007).

The purpose of this intervention was to provide the main caregivers of older dependents, who presented high emotional distress, with efficient strategies to control stress and thus, improve their emotional status. Therefore, the goals were: (a) to help caregivers to relax, using breath-control training; (b) increase their leisure time, for which a program to increase pleasant activities was carried out; (c) modify their subjective appraisal of the situation; specifically, to decrease their perception of burden and their reaction to the older person's memory and behavior problems, using cognitive restructuring; (d) increase their satisfaction with social support, using assertive communication training; (e) improve the application of coping strategies to caregiving (with a decrease in emotionfocused coping, which implicitly means an increase in problem-focused coping), using problem-solving training; and lastly, (f) improve their self-esteem, through a specific program.

When the intervention was ready, a pilot study was carried out ( $n=4$, TWS $=2$, MTC $=2$ ) to determine potential problems in its implementation. The final intervention was highly structured. In fact, a treatment handbook was elaborated. Bach session involved a brief review of the previous week's homework in order to firmly establish the skills practiced in the natural setting, followed by the development of the specific components of each session, and finishing with a summary of the material from that the session and handing out new homework. At the end of each session, the time and place for the next meeting was set. up and the material with a summary of the session was handed out. In the first session, the program was presented and the initial assessment carried out. In the last session, potential future problems were foreseen and post-treatment assessment was performed.

\section{Intervention conditions}

The intervention was carried out in two formats, one of TWSs and the other of MTC. Both intervention formats were performed individually and had the same components. The formats only differed in the intensity of therapist-patient contact and the amount of homework.

\section{Traditional weekly sessions}

This intervention format consisted of eight weekly individual sessions (total duration: 2 months), all of them in contact with the therapist and lasting about $\approx 60 \mathrm{~min}$. Diaphragmatic breathing was practiced for seven sessions (2 7); increase in pleasant activities was developed during three sessions (3 5); cognitive restructuring in three sessions (5 7); assertive communication in two sessions ( 6 and 7 ); problemsolving training in one session (4); and techniques to improve self-esteem in one session (7).

\section{Minimal therapist contact}

The duration of this format was also 2 months, consisting of three individual sessions with the therapist, each one lasting about $90 \mathrm{~min}$, with an interval of 4 weeks between sessions. During these intervals, the caregivers worked at home on the materials provided in the previous session, and they received periodical phone calls from the therapist, lasting about $10 \mathrm{~min}$. Three phone contacts were established in order to help and encourage the caregiver and to supervise the evolution of the homework. The first phone call was made between the second and third week, the next one before the fourth week, and the last one between the sixth and seventh week.

In contrast to TWS, in this intervention format, diaphragmatic breathing was introduced in the first session in order to practice and train it with the therapist. In the second session (week 5), caregivers were initiated in cognitive restructuring and assertive communication, in order to initiate the practice of these therapeutic components in the presence and under the direct supervision of the therapist. The caregivers practiced the remaining therapeutic elements at home with the heip of materials prepared to this effect.

The time, measured in weeks, dedicated to each one of the therapeutic components was the same in both formats, except for breathing control, which was practiced a week more in MTC because it was already introduced in the first session.

\section{Design}

\section{Assignation of caregivers to the} experimental conditions

Using the computer program 'Randomization.com' (http://www.randomization.com), the professionals from the institutions who had agreed to collaborate in the study (social workers, nurses, doctors, and psychologists) and who were in contact with the caregivers were randomly assigned to group MTC or group TWS. The method of randomly permuted blocks was used. Thus, if a professional was randomly assigned to group TWS, he or she could only offer this treatment format to the caregivers; on the contrary, if the professional was assigned to group MTC, only this kind of intervention was offered.

Suicide ideation was the exclusion criterion of the patients randomized to the MTC group, as is the case 
with other MTC programs, as it does not seem ethical to treat patients at risk of suicide with programs of limited or null therapist contact (Jamison \& Scogin, 1995; Woody, McLean, Taylor, \& Koch, 1999). Taking the above into account, the total sample was made up of 86 caregivers, 42 of them assigned to the TWS format and 44 to the MTC format.

\section{Measurement times}

In addition to the initial pretreatment measurement, all the participants were assessed again 2 months later, at post-treatment. Moreover, follow-up measures were carried out at $1,3,6$, and 12 months.

\section{Variables}

Treatment effects were observed in the dependent variables that reflect caregivers' emotional status (anxiety and depression), as well as in the mediating variables of such status, among which can be differentiated variables that involve caregivers' personal resources (i.e., coping strategies, social support, and self-esteem) on the one hand, and that are related to caregivers' appraisal of their situation (i.e., burden), on the other. Sociodemographic variables and variables involving the care provided were also assessed to allow us to determine the participants' characteristics and their care-giving situation. All sessions and assessments were led by the first author (J. López).

\section{Measures}

The assessment battery was made up of the following instruments:

The Interview Guide for Caregivers (Crespo \& López, 2007) collects sociodemographic information and information about the care being provided. Sociodemographic characteristics of caregivers (i.e., age, gender, kinship, work status) and of the older people (i.e., age, gender, medical diagnosis) were assessed, as well as the care situation (i.e., type of caregiver, weekly hours providing care, how long they had been caring for the relative).

The Katz Index of Activities of Daily Living (ADL; Katz et al., 1963) assesses the patient's disability in six basic self-care activities (e.g., bathing, dressing) as a measure of functional impaiment. Limitation in one to two items indicates moderate dependency, whereas limitation in three or more items is considered severe dependency. The internal consistency of the Spanish version is satisfactory, with Cronbach's $\alpha$ at 0.91 (Lzal, Montorio, Márquez, \& Losada, 2005).

The Anxiety Subscale of the Hospital Anxiety and Depression Scale (HAD-A; Zigmond \& Snaith, 1983) assesses the presence of anxiety symptoms. According to the authors of the scale, scores between 0 and 7 indicate absence of anxiety symptomatology, scores between 8 and 10 are doubtful cases, and scores between 11 and 21 indicate the presence of anxiety symptomatology. $\mathrm{A}$ recent review verified that at a cut-off point of 8 , the sensitivity and specificity of the subscale is $80 \%$. The mean $\alpha$ across these studies was 0.83 (Bjelland, Dahl, Haug, \& Neckelmann, 2002).

The Beck Depression Inventory (BDI; Beck et al., 1979) identifies the global level of depression and the changes occurring over time. The published BDI Manual (Beck \& Steer, 1993) proposes a cut-off point of $17 / 18$ to differentiate minimum or slight levels of depression from moderate or severe levels. Thus, scores over 17 are indicators of a possible depressive disorder. Administered to caregivers, it has shown a sensitivity of $70 \%$ and a specificity of $79 \%$ at a cut-off point of $9 / 10$ (slight depression) (Gallagher, Rose, Rivera, Lovett, \& Thompson, 1989).

The Burden Interview (BI; Zarit, Reever, \& BachPeterson, 1980) evaluates feelings of burden specific to an individual's role as caregiver. With Spanish samples, Martin et al. (1996) found a specificity of $84.4 \%$ and a sensitivity of $85.1 \%$ at a cut-off point of $46 / 47$, and of $93.3 \%$ (both for specificity and for sensitivity) at a cut-off point of $55 / 56$. These cut-off scores identify family caregivers at risk for psychological distress and in need of further assessment and intervention. Test-retest reliability was 0.86 , and Cronbach's $\alpha$ reached the value of 0.91 .

The Brief COPE (Carver, 1997) is a 28-item instrument that, according to a study performed with caregivers (López, Crespo, Arinero, Gómez, \& Francisco, 2004), measures three essential coping styles: problem-focused (the use of strategies aimed at managing or changing the problem that is causing distress, such as planning and active coping, positive reframing, acceptance and use of instrumental and social support), emotion-focused (using methods aimed at regulating the emotional response to the problem, such as venting, denial, self-blame, behavioral disengagement and self-distraction), and the search for transcendence (praying and avoiding making fun of the situation when dealing with the problem that causes distress). The internal reliability of the first two scales is adequate, 0.75 for problem-focused coping, and 0.65 for emotion-focused coping, whereas in the case of the search for transcendence scale, the reliability is only 0.22 (López et al., 2004). Therefore, it seemed suitable to only take the main coping scales into account when performing the analyses, that is, problem- and emotion-focused coping.

The main purpose of the version of the Social Support Questionnaire, Short Form-Revised (SSQSR; I.G.Saranson, B.R. Saranson, Shearin, \& Pierce, 1987) is to measure the availability of and satisfaction with social support. Six items were selected from the original scale upon verifying in a previous study that these items explained a sufficient quantity of the variances of the available amount of social support and satisfaction with such support, and they presented an internal consistency of 0.55 for available support and 0.72 for satisfaction with support (López, 2005). Test-retest reliability of the short form was 0.90 for 
available support and 0.83 for satisfaction with support (Saranson et al., 1987).

The Rosenberg Self-Esteem Scale (RSE; Rosenberg, 1965 ) assesses, with 10 items, the levels of caregivers' self-esteem, that is, a person's feelings of selfsatisfaction and self-acceptance. Its internal consistency is 0.81 and its discriminant validity is adequate; thus, for example, the scale has a significant and negative correlation of -0.35 with the BDI (Arinero, Crespo, López, \& Gómez, 2004).

The complete assessment battery was administered at pretreatment. At post-treatment and at the 6- and 12 -month follow-ups, all the measuring instruments, except for those concerning sociodemographic data, were again administered. At the 1- and 3-month follow-ups, only the HAD-A and the BDI were administered in order to avoid as much as possible caregivers' fatigue and their dropping out of the investigation.

\section{Data analysis}

Missing data, as long as it did not exceed $15 \%$ of the total self-report, was replaced by the data provided by these same subjects in the immediately preceding measurement.

Likewise, intention-to-treat analysis was performed, so that the data of all the caregivers who initiated treatment and who participated in more than $25 \%$ of the sessions were included in the analysis of treatment efficacy. That is, any subject who participated in more than two sessions was included in the analyses. Thus, in the case of dropouts after the second session, all their data at their last recorded measurement time was maintained at post-treatment and follow-up. Pretreatment data were maintained if caregivers dropped out without concluding the treatment and the last follow-up data were maintained if they dropped out after concluding the treatment.

Chi-square and ANOVAs for independent measures were used to verify the homogeneity of the groups. To analyze the changes produced over time and the differences between the two intervention formats, a repeated measures' ANOVA was conducted for each of the variables analyzed, taking into account a between-subject factor (two levels: traditional format and minimal contact) and a within-subject factor (six levels for the variables anxiety and depression and four levels for the rest of the variables studied). The sphericity assumption was verified by the Mauchly test. On all occasions, Bonferroni's correction was used for multiple comparisons and, when the sphericity assumption was not met, we used the Huynh Feldt correction. Paired comparisons were obtained in order to determine the mean differences of the participants between pretreatment and each one of the subsequent measurement times.

Moreover, one-factor (intervention format) ANCOVAs of each of the variables analyzed were carried out, with the pretreatment assessment value as covariate, for the diverse post-treatment and follow-up measurements. Thus, the differences between the two intervention formats at each measurement time were obtained, while controlling the effects of the pretreatment values.

Lastly, regarding the clinical significance of the results, McNemar tests were performed to determine the pre post changes in the percentages of caregivers with scores over the cut-off point for anxiety and depression in the two intervention formats, and the relative risk and the effect size were calculated.

\section{Results}

\section{Adherence to treatment}

There were five dropouts, three before ending treatment (one because the elderly relative died and two because the relatives were institutionalized) and the remaining two dropouts occurred at the 12-month follow-up (one due to death, and one to institutionalization). As treatment was individual, thus allowing us to set up a suitable schedule for the meetings, dropouts were very scarce. It seems that participants only dropped out of the intervention formats due to causes of 'force majeure'. Of the five dropouts, four occurred in the TWS format, which apparently indicates worse adherence to treatment in this format, but it should not be forgotten that compliance with the homework assignments also reveals the caregivers' adherence to the intervention formats. In fact, attending a session did not guarantee that they would do the homework. A significantly higher number of homework assignments were completed by participants who were in the TWS format $(M=12.88)$ versus the MTC format $(M=6.46), F(1,78)=36.977$, $p<0.001$ ).

\section{Sample characteristics and group homogeneity}

Most of the 86 caregivers who began treatment were women $(89.5 \%)$, the older relative's children $(62.85 \%)$, and did not work out of the home $(52.2 \%$ ) (i.e., they were housewives, retired, or unemployed), and their age ranged between 29 and 80 years $(M=53.30$, $\mathrm{SD}=11.31$ ).

Women not only largely predominated among the population that provided informal help, but also among those who received such help $(69.8 \%)$. The receivers of informal help had a mean age of 77.59 years $(\mathrm{SD}=8.35)$, with a range between 60 and 95 years. Most of them $(80.2 \%)$ presented a diagnosis of dementia.

Caring for an elderly dependent is a very demanding task. Thus, the caregivers dedicated an average of $97.82 \mathrm{~h}$ per week $(\mathrm{SD}=60.75)$, that is, about $14 \mathrm{~h}$ per day, and they had played this role for an average of 66 months ( $\mathrm{SD}=77.17$ ), that is 5.5 years. It should not be forgotten that the elderly people needed help or 
supervision for an average $3.67(\mathrm{SD}=1.71)$ activities of daily living (ADLs). Caregivers considered themselves to be the elderly person's main caregiver $(76.7 \%)$ or the only caregiver of their family $(23.3 \%)$.

The randomization process was successful as there were no significant pretreatment differences between the caregivers of the TWS and the MTC formats in any of the variables studied.

\section{Treatment efficaey}

Dependent variables

As can be seen in Table 1, the variable anxiety showed significant within-subject differences. In fact, significant differences were produced between the pretreatment means and all the subsequent measurement times. As can be seen in Table 2 and in Figure 1,

Table 1. Analysis of the differences in the dependent variables at various measurement times $(n=86)$.

\begin{tabular}{|c|c|c|}
\hline Variables & $F_{(\mathrm{da})} / \mathrm{kan}$ & Significance \\
\hline \multicolumn{3}{|l|}{ Anxiety } \\
\hline Within-subjects factor (Time) & $F_{(4, A 88,363.563)}=16.767^{\mathrm{e}}$ & $p<0.001$ \\
\hline $\begin{array}{l}\text { Within-subjects comparisons } \\
\text { Pre-post }\end{array}$ & 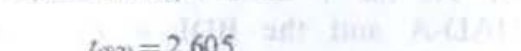 & \\
\hline $\begin{array}{l}\text { Pre-post } \\
\text { Pre-1-month follow-up }\end{array}$ & $\begin{array}{l}t_{(82)}=2.605 \\
t_{(s 2)}=3.551\end{array}$ & $\begin{array}{l}p<0.001 \\
p<0.001\end{array}$ \\
\hline Pre-3-month follow-up & 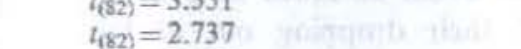 & $\begin{array}{l}p<0.001 \\
p<0.001\end{array}$ \\
\hline Pre-6-month follow-up & $t_{(82)}=2.418$ & $p<0.001$ \\
\hline Pre-12-month follow-up & $t_{(82)}=2.845$ & $p<0.001$ \\
\hline \multicolumn{3}{|l|}{ Depression } \\
\hline Within-subjects factor (Time) & $F_{(3,771,305.431)}=16.243^{2}$ & $p<0.001$ \\
\hline Within-subjects comparisons ${ }^{6}$ : & & \\
\hline a & $t_{(8 z)}=5.428$ & $p<0.001$ \\
\hline Pre-1-month follow-up & 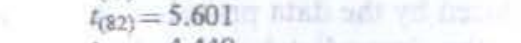 & $p<0.001$ \\
\hline Pre-3-month follow-up & 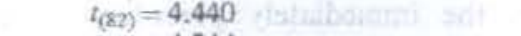 & $p<0.091$ \\
\hline Pre-6-month follow-up & $t_{(82)}=4.244$ & $p<0.001$ \\
\hline Pre-12-month follow-up & $t_{(82)}=5.158$ & $p<0.001$ \\
\hline
\end{tabular}

Notes: "Huynh-Feldt correction factor was used.

${ }^{b}$ Bonferroni correction factor was used.

Table 2. Evolution of the results of the dependent and mediating variables $(n=86)$.

\begin{tabular}{|c|c|c|c|c|c|}
\hline \multirow[b]{2}{*}{ Variables } & \multicolumn{5}{|c|}{ Time of measure but sil tail tor } \\
\hline & Pre-treatment & Post-treatment & 1 Month & 3 Months & 12 Months \\
\hline \multicolumn{6}{|c|}{ Anxiety (range 0-21) } \\
\hline TWS & $11.51(3.97)$ & $7.73(3.27)$ & $7.22(3.32)$ & $8.39(4.68)$ & $7.56(4.03)$ \\
\hline MTC & $10.74(3.63)$ & $9.31(3.95)$ & $7.93(391)$ & $9.02(4.19)$ & $9.00(4.69)$ \\
\hline \multicolumn{6}{|c|}{ Depression (range 0-63) } \\
\hline TWS & $17.29(9.19)$ & $8.32(7.84)$ & $8.80(6.58)$ & $10.76(8.71)$ & $10.05(7.68)$ \\
\hline MTC & $13.29(7.52)$ & $11.40(7.46)$ & $10.57(8.48)$ & $11.38(7.69)$ & $10.21(8.00)$ \\
\hline \multicolumn{6}{|c|}{ Burden (range $28-112$ ) } \\
\hline TWS & $64.34(12.95)$ & $57.05(12.48)$ & - & $6(17-+6)=60.71(13.11)$ & $58.12(12.32)$ \\
\hline MTC & $67.12(13.67)$ & $62.81(13.67)$ & - & 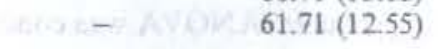 & 14.82) \\
\hline \multicolumn{6}{|c|}{ Problem-focused coping (range 1-4) } \\
\hline TWS & $2.45(0.58)$ & $2.79(0.49)$ & - & $2.53(0.59)$ & $2.45(0.58)$ \\
\hline MTC & $2.29(0.56)$ & $2.41(0.53)$ & - & $-\quad 2.22(0.56)$ & $2.27(0.49)$ \\
\hline \multicolumn{6}{|c|}{ Emotion-focused coping (range 1-4) } \\
\hline TWS & $1.82(0.44)$ & $1.80(0.34)$ & - & $1.78(0.46)$ & $1.71(0.38)$ \\
\hline MTC & $1.66(0.47)$ & $1.61(0.38)$ & - & $1.66(0.37)$ & $1.62(0.46)$ \\
\hline \multicolumn{6}{|c|}{ Self-esteem (range 10-40) } \\
\hline TWS & $28.32(4.58)$ & $30.24(3.48)$ & - & $-\quad 29.24(4.08)$ & $30.02(4.29)$ \\
\hline MTC & $29.00(3.53)$ & $29.83(3.55)$ & - & $\begin{array}{l}-\quad 30.19(3.96) \\
\end{array}$ & $31.19(3.91)$ \\
\hline \multicolumn{6}{|c|}{ Social support (range 0-18) } \\
\hline TwS & $5.92(4.46)$ & $9.98(5.63)$ & - & $-(16.578 \quad 8.66(5.73)$ & $9.66(6.22)$ \\
\hline MTC & $5.83(4.49)$ & $7.29(4.79)$ & - & $-145=7.28(4.83)$ & $6.26(3.77)$ \\
\hline \multicolumn{6}{|c|}{ Satisfaction with social support (range 3-18) } \\
\hline TwS & $11.85(3.21)$ & $14.97(3.36)$ & - & $14.58(3.61)$ & $14.51(3.26)$ \\
\hline MTC & $12.45(4.13)$ & $13.67(3.94)$ & - & $13.57(2.94)$ & $13.50(3.05)$ \\
\hline
\end{tabular}

Note: TWS (traditional weekly sessions) and MTC (minimal therapist contact) are shown as $M$ (SD). 
the means of anxiety show a pronounced decrease between the baseline or pretreatment value and the post-treatment value and these improvements are sustained at the follow-ups of both intervention formats.

As can be seen in Table 3, no significant between-subject differences were obtained in anxiety or in the Time $x$ Intervention interaction. However, the diverse ANCOVAs reveal the existence of significant differences in this variable between the intervention formats at post-treatment and at the 3- and 12-month follow-ups.

In the case of depressed mood, significant withinsubject differences were observed, In fact, there were significant differences between the pretreatment means and all the subsequent measurements (Table 1).

Significant differences were also obtained for the Time $x$ Intervention interaction, but no significant between-subject differences were obtained (Table 3 ).

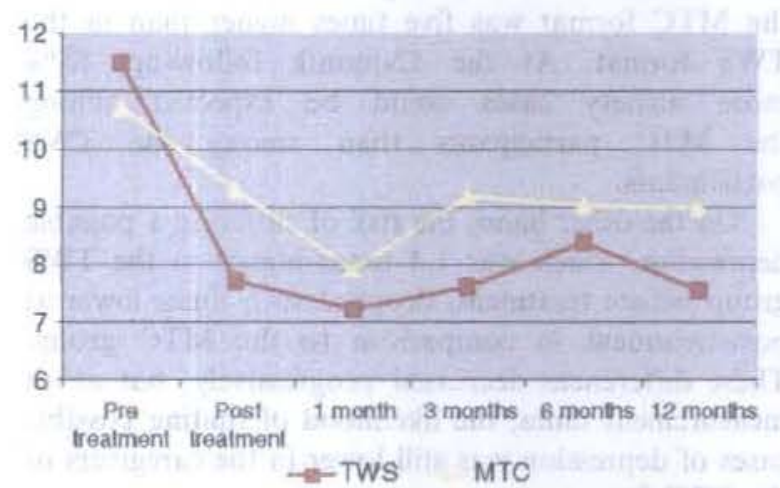

Figure 1. Evolution over time of the levels of anxiety (Anxiety Subscale of the Hospital Anxiety and Depression Scale) in the two experimental groups $(n=86)$.
As can be seen in Table 2 and Figure 2, the means show an important post-treatment decrease compared to pretreatment, and these levels remained relatively stable at the follow-ups in the TWS intervention format, whereas the decrease was more progressive in the MTC format. The diverse ANCOVAs revealed significant differences in depression between the two intervention formats at post-treatment and at the 1-month follow-ip.

\section{Mediating variables}

Significant within-subject differences were observed in burden, $F(2.389,193.492)=10.966, p<0.001$, with a decrease in the participants' levels of burden over time. Mean comparisons indicated significant reductions between pretreatment and all the subsequent measurement times: post-treatment, $t(82)=5.801 ; p<0.001$, 6-month follow-up, $t(82)=4.519 ; \quad p<0.017$, and

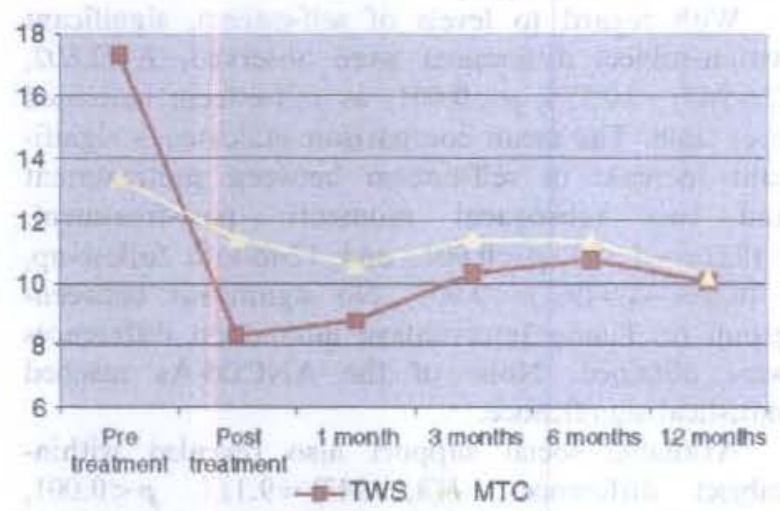

Figure 2. Evolution over time of the levels of depression (Beck Depression Inventory) in the two experimental groups $(n=86)$.

Table 3 . Analysis of the differences between the two experimental groups with the dependent variables at the varions measurement times $(n=86)$.

\begin{tabular}{|c|c|c|}
\hline 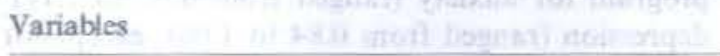 & $F_{(a)}$ & Significance \\
\hline Anxiety & & \\
\hline Between-subjects factor (intervention) & $F_{(1,81)}=1.421$ & n.s. \\
\hline $\begin{array}{l}\text { Time } x \text { Intervention interaction } \\
\text { ANCOVAS }\end{array}$ & $F_{(4.448,363.563)}=2.303^{2}$ & n.s. \\
\hline Post-treatment & $=10.99$ & $p<0.001$ \\
\hline 1-month follow-up & $F_{(1,80)}=2.12$ & n.s. \\
\hline 3-month follow-up & $F_{(1,80)}=4.21$ & $p<0.042$ \\
\hline 6-month follow-up & $F_{(1,80)}=1.23$ & \\
\hline 12-month follow-up & $F_{(1,80)}=4.03$ & $p<0.048$ \\
\hline Depression & & \\
\hline Between-subjects factor (Intervention) & $F_{0.81}=0.90$ & n.s. \\
\hline Time $x$ Intervention Interaction & $P_{(3.77,305.431)}=5.341^{2}$ & $p<0.001$ \\
\hline 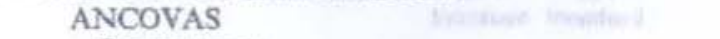 & 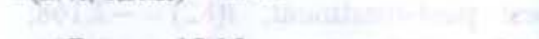 & \\
\hline Postreatment & $F_{(1,80)}=12.15$ & $p<0.001$ \\
\hline 1-month follow-up & $F_{(1.80)}=5.26$ & $p<0.024$ \\
\hline 3-month follow-up & $F_{(1,80)}=2.94$ & n.s. \\
\hline 6-month follow-up +1 & $F_{(1,80)}=2.15$ & n.s. \\
\hline 12-month follow-up & 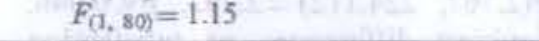 & n. \\
\hline
\end{tabular}

Note: "Huynh-Feldt correction factor was used. 
12-month follow-up, $t(82)=6.776 ; p<0.001$. No significant between-subject or Time $x$ Intervention interaction differences were found. Likewise, none of the ANCOVAs reached statistical significance.

Regarding coping strategies, problem-focused coping showed significant within-subject differences, $F(3,243)=8.156, \quad p<0.001$, with an increase in participants' use of this kind of coping. Mean comparisons indicated significant increases between pre- and post-treatment, $t(82)=-0.228 ; p<0.001$. This kind of coping was also being used significantly more in the participants of the TWS format, $F(1,81)=6.758, p<0.011$, than in the MTC format, but the differences in the interaction Time $x$ Intervention were not statistically significant. More frequent use of this coping was also observed in the TWS format than in the MTC format at posttreatment, $F(1,80)=10.761, p<0.002$, and at the 6-month follow-up, $F(1,80)=4.259, \quad p<0.042$. However, there were no significant differences in emotion-focused coping.

With regard to levels of self-esteem, significant within-subject differences were observed, $F(2.802$, $226.948)=10,576, p<0.001$, as self-esteem increased over time. The mean comparison indicated a significant increase in self-esteem between pretreatment and two subsequent moments: post-treatment, $t(82)=-1.380 ; p<0.001$, and 12 -month follow-up, $t(82)=-1.949 ; p<0.001$. No significant betweengroup or Time $x$ Intervention interaction differences were obtained. None of the ANCOVAs reached statistical significance.

Available social support also revealed withinsubject differences, $F(3,243)=9.111, p<0.001$, increasing over time. Mean comparisons indicated significant increases between pretreatment and all the other subsequent measurement times: post-treatment, $t(82)=-2.751 ; \quad p<0.001,6$-month follow-up, $t(82)=-2.092 ; p<0.001$, and 12 -month follow-up, $t(82)=-2.080 ; p<0.007$. Social support was significantly higher in the TWS format than the MTC format, reaching significant between-subject, $F(1,81)=4.727), p<0.033$, and Time $\times$ Intervention interaction, $F(3,243)=3.402, p<0.018$, differences. At post-treatment, more social support was observed among the caregivers in the TWS format, $F(1,80)=6.87, p<0.011$, in comparison with the MTC format.

Participants' satisfaction with social support showed significant within-subject differences, $F(2.767,224.112)=14.878, p<0.001$, increasing over time. The mean comparisons indicated significant increases between pretreatment and all the subsequent measurement times: post-treatment, $t(82)=-2.168$; $p<0.001, \quad 6$-month follow-up, $\quad t(82)=-1.925$; $p<0.001$, and 12-month follow-up, $t(82)=-1.853$; $p<0.001$. Time $\times$ Intervention interaction differences were obtained, $F(2.767,224.112)=2.792, p<0.046$, but not between-subject differences, as satisfaction increased in the TWS format, whereas it remained stable after a slight post-treatment increase in the MTC format. Significantly, higher satisfaction was found in the TWS format at post-treatment, $F(1,80)=7.703$, $p<0.007$, and at the 12 -month follow-up, $F(1,80)=$ $4.094, p<0.046$, in comparison to the MTC format.

\section{Clinical significance}

From the clinical viewpoint, the MeNemar tests showed a significant reduction in the percentages of possible cases of anxiety and depression between pretreatment and each and every one of the subsequent measurement times.

As observed in Figure 3, there were important improvements in emotional status, with significant reductions in the relative risk of possible anxiety and depression problems in the TWS format. The risk of anxiety problems was similar in both formats before treatment and decreased among those who participated in the TWS format. Thus, at post-treatment, the likelihood of finding anxiety disorders in the MTC format was five times higher than in the TWS format. At the 12-month follow-up, $63 \%$ more anxiety cases could be expected among the MTC participants than among the TWS participants.

On the other hand, the risk of suffering a possible depression, which was 1.4 times higher in the TWS group before treatment, dropped to 5 times lower at post-treatment in comparison to the MTC group. These differences decreased progressively, but at all measurement times, the likelihood of finding possible cases of depression was still lower in the caregivers of the TWS format.

In order to quantify the clinical impact of the intervention, the effect size for each dependent variable was determined, classifying them according to the values proposed by Faul and Erdfelder (1992) (i.e., small $<0.5$; medium $>0.5$ and $<0.8$; and large $>0.8$ ). Large effect sizes were found in the TWS treatment program for anxiety (ranged from 0.95 to 1.17 ) and depression (ranged from 0.84 to 1.06 ), except for the

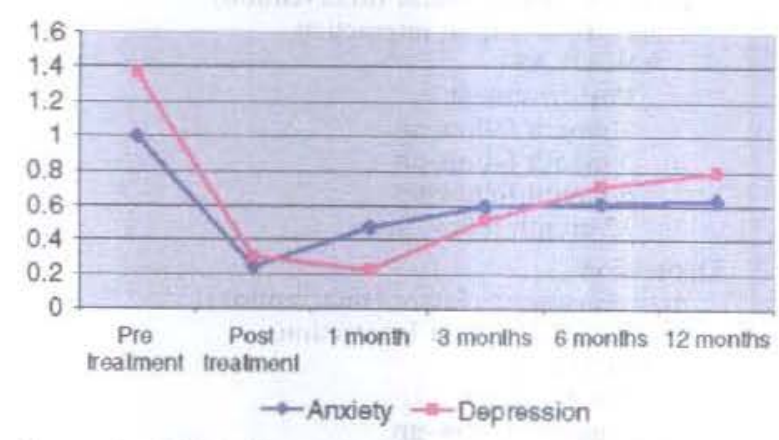

Figure 3. Risk indices of the presence of possible anxiety (Anxiety Subscale of the Hospital Anxiety and Depression Scale $>10$ ) and depression disorders (Beck Depression Inventory $>17$ ) in the TWS intervention gromp vs. the MTC gronp at various measurement times $(n=86)$. 
6-month follow-up when the effects sizes were medium ( 0.71 and 0.72 , respectively). However, the effect sizes were small for the MTC intervention (ranged from 0.23 to 0.43 ), except for the 1 -month follow-up for anxiety when it was medium $(0.74)$.

\section{Discussion}

This investigation performs a detailed analysis of the efficacy of a treatment program, with two formats, to improve informal caregivers' emotional status, using for this purpose rather restrictive selection criteria: a large sample of caregivers, a meticulous assessment, and sufficiently extended follow-ups. Moreover, it complies with most of the prerequisites that investigations of treatment efficacy should meet (Seligman, 1995): (a) random assignment of patients to the experimental conditions, (b) in-depth assessment, (c) clearly described and protocolized treatments, (d) a fixed number of sessions, and (e) long-term follow-up of patients.

The data show that the stress-management training proposed herein (both intervention formats) contributes to a decrease in the initial levels of anxiety, depression, even improving the levels of perceived burden, problem-focused coping, social support, and levels of self-esteem. In fact, previous data have shown that caregivers receiving this stress-control program had a greater reduction in anxiety and depressive symptoms immediately following the intervention than did the people in an untreated control group (Lopez, Crespo \& Zarit, 2007).

Therefore, it seems necessary to continue to offer such cognitive-behavioral psychotherapeutic interventions to people who are emotionally affected to help them reduce their levels of distress. According to research and the theoretic models, there are two elements that affect caregivers' emotional problems decisively. First personal resources (i.e., levels of coping, social support, and self-esteem), and second, their appraisal of their situation. The present stressmanagement intervention improved these mediating variables (e.g., problem-focused coping, available support, satisfaction with social support, and levels of self-esteem increased, and the perception of burden decreased) and perhaps, that is why it was effective.

It would be too pretentious to expect to modify such a multiple and complex problem as the chronic stress suffered by many caregivers of elderly dependent people by means of a single and brief intervention (Mahoney et al., 2003). Therefore, a more reasonable clinical goal could be to attempt to stabilize their emotional problems (López \& Crespo, 2007). The intervention herein described managed to reduce important aspects of caregivers' emotional problems (especially their levels of anxiety and depression) without being able to modify others substantially. In some conditions, if problems or their impact on the person do not increase, it is considered a therapeutic achievement. Such is the case of the caregivers of elderly dependents, who endure many problems and difficulties, generally of increasing intensity and quantity. Thus, the substantial improvements achieved in this intervention in some variables make special relevance. This could have to do, at least partially, with the fact that the intervention was administered exclusively to caregivers with emotional problems (which is not very common in this kind of studies). This may have compensated for the 'floor effects' that may occur in persons who present initially low levels of anxiety and depression.

The refusal and dropout rates of the treatment proposed herein were very low, which is surprising, as in the works about caregivers, the percentages of dropouts and refusals is usually high (López \& Crespo, 2007; Sörensen et al., 2002). This good result may be related to the flexibility and the individual nature of the program, and also to the fact that it targeted people who really needed it (that is, who showed considerable emotional distress). The fact that the dropouts of the stress-management program are not very numerous $(5.6 \%)$ can be considered a success. But it should not be forgotten that to attend a session did not guarantee performing the homework, as only $53 \%$ of the assigned homework was performed.

Moreover, in some aspects, it is notable that the TWS intervention format presents different results from those of the MTC format. For instance, the TWS format reduced the levels of anxiety and depression more than the MTC format, in addition to the fact that, on the one hand, it achieved higher levels of caregivers' problem-focused coping and, on the other, the caregivers had more available support and felt more satisfied with such support. In any case, both intervention formats showed a different change tendency. In the TWS format, the reduction of emotional distress occurred essentially between preand post-treatment, and these improvements tended to be sustained at follow-ups. In contrast, in the MTC format, the reduction tended to be progressive. It therefore seems that learning and putting into practice the strategies proposed by the stress-management program was carried out quickly, almost immediately, by the caregivers in the TWS format, whereas the caregivers in the MTC format needed more time to assimilate and put these strategies into practice. This may be because in the TWS interventions, there was, on the one hand, more contact, and more work in the treatment phase in comparison to the MTC group. Moreover, there was such an important reduction between pre- and post-treatment that it was difficult to continue decreasing to any further extent the levels of the emotional problems they suffered.

Thus, there are some differences between the intervention formats, both in the general efficacy of the programs and in the evolution of the dependent and mediating variables. However, one wonders whether the differences found 'compensation' for the additional effort required from the caregiver who 
participates in a program of weekly sessions, and whether, there are different profiles of caregivers who could benefit from one format or the other.

We must acknowledge a series of limitations that should be taken into account when interpreting the results: (1) the evaluations were not performed by blind interviewers; (2) the randomization was carried out at first on the professionals instead of on the caregivers. This was because of the fact that it is difficult to access the caregivers unless the professionals with whom they are in contact tell them what kind of intervention is being offered by the therapist. The caregivers and the professionals from the diverse institutions are usually averse to the practice of randomization. However, the possible biases that may have been introduced were not particularly relevant as it was shown by the homogeneity analysis; and (3) despite the fact that we attempted to plan a simple treatment, that is, with materials of easily understandable content, simple homework, self-reports that were easy to fill in ... actually, the intervention may be somewhat difficult for caregivers of a low educational level, who are not used to reading and writing. Some of the therapeutic components were developed orally in session, but others were developed in writing during the session and at home, and this may be a limitation for the implementation of the intervention.

As can be seen, the intervention described herein is not a special extensive stress-management training program. Nevertheless, an 8-session treatment, such as the one tested here, would be rated as medium high intensity, according to the meta-analysis of Knight et al. (1993). The number of sessions could be increased in order to spend more time working on each of the stress-management techniques proposed but this would probably increase the dropouts and also, particularly, the number of refusals to participate in the intervention.

Lastly, all the efforts carried out with this investigation, independently of the results and limitations, were aimed at offering the caregivers, with patience and understanding, the possibility of learning to manage the stress they endure, as much as possible.

\section{Acknowledgements}

This research was supported by a grant from the Complutense University of Madrid, Spain, to the first author. Our thanks to all the caregivers involved in this study and also to their institutions, and to the professionals who spread word of the intervention.

\section{References}

Arinero, M., Crespo, M., López, J., \& Gómez, M. (2004). Analysis of Rosenberg self-esteem scale: Reliability and validity of items. Proceedings of the 7th Enropean Conference on Psychological Assessment (p. 116). Malaga, Spain.
Beauchamp, N., Irvine, A.B., Seeley, J., \& Johnson, B. (2005). Worksite-based Internet multimedia program for family caregivers of persons with dementia. Gerontologist, 45, 793-801.

Beck, A.T., Rush, A.J., Shaw, B.F., \& Emery, G. (1979). Cognitive therapy of depression. New York: Guilford Press.

Beck, A.T., \& Steer, R.A. (1993). Beck Depression Inventory. Manual. San Antonio. TX: The Psychological Corporation.

Bjelland, L, Dahl, A.A., Hang, T.T., \& Neckelmann, D. (2002). The validity of the Hospital and Depression Scale. Joumal of Psychosomatic Research, 52, 69-77.

Brodaty, H., Green, A., \& Koschera, A. (2003). Metaanalysis of psychosocial interventions for caregivers of people with dementia. Journal of the American Geriatric Saciety, 51, 657-664.

Carver, C.S. (1997). You want to measure coping but your protocol's too long: Consider the Brief Cope. International Joumal of Behavioral Medicine, 4, 92-100.

Chang, B.L. (1999). Cognitive-behavioral intervention for homebound caregivers of persons with dementia. Nursing Research, 48, 173-182.

Cooke, D.D., MeNally, L., Mulligan, K.T., Harrison, MJ.G., \& Newman, S.P. (2001). Psychosocial interventions for caregivers of people with dementia: A systematic review. Aging and Mental Health, 5, 120-135.

Crespo, M., \& López, J. (2007). El estrés en cuidadores de mayores dependientes [Stress in caregivers of elder dependents]. Madrid: Pirámide.

Crespo, M., López, J., \& Zarit, S. (2005). Depression and anxiety in primary caregivers: A comparative study of caregivers of demented and nondemented older persons. International Joumal of Geriatric Psychiatry, 20, 591-592.

Cuijpers, P. (2005). Depressive disorders in caregivers of dementia patients: A systematic review. Aging and Menial Heaith, 9, 325-330.

Davis, L.L., Burgio, L.D., Buckwalter, K.C., \& Weaver, M. (2004). A comparison of in-home and telephone-based skill training interventions with caregivers of persons with dementia. Joumal of Mental Health and Aging, 10,31-44.

Eisdorfer, C., Czaja, S.J., Loewenstein, D.A., Rubert, M.P. Arguelles, S., Mitrani, V.B., et al. (2003). Effect of family therapy and technology-based intervention on caregiver depression. Gerontologist, 43, 521-531.

Faul, F., \& Erdfelder, E. (1992). GPOWER. Bonn: Bonn University.

Gallagher, D.E. (1985). Intervention strategies to assist caregivers of frail elders. In M.P. Lawton, \& G. Maddox (Eds.), Annual review of gerontology and geriatrics (Vol, 5, pp. 249-282). New York: Springer.

Gallagher, D., Rose, J., Rivera, P., Lovett, S., \& Thompson, L.W. (1989). Prevalence of depression in family caregivers. Gerontologist, 29, 449-456.

Gould, RA., \& Clum, G.A. (1993). A meta-analysis of selfhelp treatment approaches. Clinical Psychology Review, 13, 169-186.

Izal, M., Montorio, I., Márquez, M., \& Losada, A. (2005). Caregivers' expectations and care receivers competence. Archives of Gerontology and Geriatrics, 41, 129-140.

Jamison, C., \& Scogin, F. (1995). The outcome of cognitive bibliotherapy with depressed adults. Journal of Consulting and Clinical Psychology, 57, 403-407.

Kat2, S., Ford, A.B., Moskowitz, R.W., Jackson, B.A., \& Jaffe, M.W. (1963). Studies of illness in the aged. The index of ADL. Joumal of the American Medical Asssociation, 185, 914-919. 
Knight, B.G., Lutzky, S.M., \& Macofsky-Urban, F. (1993). Meta-analytic review of interventions for caregiver distress. Gerontologist, 33, 240-248.

López, J. (2005). Entrenamiento en manejo del estrés en cuidadores de familiares mayores dependientes [Stress management intervention for primary caregivers of dependent dwelling elder relatives] Unpublished doctoral dissertation. Universidad Complutense de Madrid, Spain.

López, J., \& Crespo, M. (2007). Intervenciones con cuidadores de familiares mayores dependientes [Interventions for caregivers of older and dependent adults]. Psicothema, 19, 72-80.

López, J., Crespo, M., Arinero, M., Gómez, M.M., \& Francisco, N. (2004). Initial analysis of psychometric properties of the Brief-COPE in a sample of caregivers. In European Association on Psychological Assessment, Proceedings of the 7th European Conference on Psychological Assessment (p. 205). Malaga, Spain.

López, J., Crespo, M., \& Zarit, S. (2007). Assessment of the efficacy of a stress management program for informal caregivers of dependent older persons. Gerontologist, 47, 205-214.

López, J., López-Arrieta, J., \& Crespo, M. (2005). Factors associated with the positive impact of caring for elderly and dependent relatives. Archives of Gerontology and Geriatrics, 41, 81-94.

Mahoney, D.F., Tarlow, B.J., \& Jones, R.N. (2003). Effects of an antomated telephone support system on caregiver burden and anxiety: Findings from the REACH for TLC intervention study. Gerontologist. 43, 556-567.

Martín, M., Salvado, I., Nadal, S., Miji, L.C., Rico, J.M., Lanz, P., et al. (1996). Adaptación para muestro medio de la escala de sobrecarga del cuidador de Zarit [Adaptation for our context of the Zarit Caregiver Burden Interview]. Revista de Gerontologia, 6, 338-346.
Marziali, E., \& Donahue, P. (2006). Caring for others: Internet video-conferencing group intervention for family caregivers of older adults with neurodegenerative disease. Gerontologist, 46, $398-403$.

Pusey, H., \& Richards, D. (2001). Systematic review of the effectiveness of psychosocial interventions for carers of people with dementia. Aging and Mental Health, 5, 107-119.

Rosenberg, M. (1965). Society and the adolescent self-image. Princeton: Princeton University Press.

Rowan, A.B., \& Storey, J.M. (2002). Minimal therapist contact. In M. Hersen, \& W. Sledge (Eds.), Encyclopedia of psychotherapy (pp. 141-145). San Diego, CA: Academic Press.

Saranson, LG., Saranson, B.R., Shearin, E.N., \& Pierce, G.R. (1987). A brief measure of social support: Practical and theoretical implications. Joumal of Social and Personal Relationships, 4, 497-510.

Schul2, R., O'Brien, A.T., Bookwala, J., \& Fleissner, K. (1995). Psychiatric and physical morbidity effects of dementia caregiving. Gerontologist, 35, 771-791.

Seligman, M.E.P. (1995). The effectiveness of psychotherapy: The Consumer Reports study. American Psychologist, 50, 965-974.

Sörensen, S., Pinquart, M., \& Duberstein, P. (2002). How effective are interventions with caregivers? An updated meta-analysis. Gerontologist, 42, 356-372.

White, M.H., \& Dorman, S.M. (2000). Online support for caregivers. Analysis of an Internet Alzheimer mailgroup. Computers in Nursing. 18, 168-176.

Woody, S., McLean, P.D., Taylor, S., \& Koch, W J. (1999). Treatment of major depression in the context of panic disorder. Joumal of Affective Disorders, 53, 163-174.

Zarit, S.H., Reever, K.E., \& Bach-Peterson, J. (1980). Relatives of the impaired elderly: Correlates of feelings of burden. Gerontologist, 20, 646-655.

Zigmond, A.S., \& Snaith, R.P. (1983). The Hospital Anxiety and Depression Scale. Acta Psychiatrica Scandinavica, 67, 361-370. 